\title{
Two kinds of adaptation in the constancy of visual direction and their different effects on the perception of shape and visual direction
}

\author{
HANS WALLACH \\ Swarthmore College, Swarthmore, Pennsylvania 19081 \\ and \\ JOSHUA BACON \\ Tufts University, Medford, Massachusetts 02155
}

\begin{abstract}
Adaptation in the constancy of visual direction can be obtained under two radically different conditions, called eye-movement adaptation and field adaptation. Adaptation resulting from these conditions and from a "normal" condition was measured with a newly developed estimation test. Eye-movement adaptation was found to cause an alteration of compensatory eye movements. It apparently consists of a changed evaluation of eye movements, as demonstrated by two different pointing tests. A form test where the shape of a large oblong is set to look square also confirmed this interpretation. After field adaptation, a pointing test did not register a change, but an adaptation effect could be measured with a forward direction test. This test and a square test where no eye movements were permitted proved to be specific to field adaptation; they measured no effect after eye-movement adaptation. The normal adaptation condition was apparently equivalent to the eye-movement adaptation condition. Its effect could be measured only with a pointing test. When we changed the normal adaptation condition so that frequent saccades were made during head turning, strong effects were measured with the two tests that were specific to field adaptation.
\end{abstract}

Those of our movements that alter the position of our eyes or our head relative to the environment cause a variety of visual stimulations which have one thing in common: they do not result in perceptions. For some reason, stimulation does not have its normal effect and the motions that it represents are not perceived. In most of these cases, it has been possible to show that the motion perceptions are prevented by specific compensation processes, each with characteristics of its own. In some instances, the result of such a compensation process has been called a constancy. The constancy of visual direction is an example. Here the displacement of the visual environment relative to the eye that is caused by turning or nodding the head is not perceived (for references see Wallach, Yablick, \& Smith, 1972). There is also a compensation for the displacement of the environment relative to the moving eye (Mack, 1970; Whipple \& Wallach, Note 1) and a compensation for the changing orientation of the environment in relation to the head caused by a tilting of the head from side to side (Wallach \& Bacon, 1976).

When one moves forward, objects that are located

This work was supported by Grant 11089 from the National Institute of Health to Swarthmore College, Hans Wallach, principal investigator. to the side of the motion path undergo partial rotations relative to the eyes, and these rotations are not seen; Wallach, Stanton, and Becker (1974) demonstrated that this was due to a compensation process. Finally, objects toward which one moves produce expanding retinal images. Wallach and Flaherty (1975) demonstrated the operation of an agent that prevents such expanding retinal images from having their normal effect on motion perception. No attempt, however, was made to determine whether the agent is a compensation process or a simple blocking effect.

How can one distinguish compensation from simple blocking? (We continue to use the constancy of visual direction as an example.) The displacement of the visual environment relative to the eyes that is caused by a turning of the head may not be perceived because all lateral displacements of the environment that take place during a turning of the head would be prevented from being perceived; any stimulation that is produced by a lateral displacement of any amount, provided it is simultaneous with a head turning, may be blocked. Compensation would have different consequences. Normally, head turning causes an angular displacement of the stationary environment relative to the head that is in the direction opposite to the head rotation and of equal amount, and this angular displacement does 
not produce perceived motion of the environment. But if one provided conditions where the angular displacement of the environment were larger or smaller than the rotation angle of the head, would these noncommensurate displacements be perceived? If all of them would go unnoticed, we would deal with a blocking effect, but this turns out not to be true. Those that are considerably larger or smaller than the displacement of a stationary environment produced by head turning will indeed be perceived. Introducing such altered displacements by having subjects wear magnifiers of small power or minifiers will cause an apparent swinging of the visual environment (Wallach \& Kravitz, 1965, 1968).

The accuracy with which compensation operates here was first investigated by Wallach and Kravitz (1965). They built an apparatus that, attached to the subject's head, put the subject's visual field under control of his head movements. That part of the subject's environment which he was allowed to see became laterally displaced when he turned his head, either in the same direction as the head rotation or in the direction against it. The rate of the environmental displacement in relation to the rate of the head rotation could also be varied, and this ratio of environmental displacement angle over headrotation angle, called displacement ratio, is what Wallach and Kravitz measured. They determined at what displacement ratios subjects reported environmental motions correctly and at what range of displacement ratios environmental motions were not perceived. They found that very small objective displacements of a luminous spot, which, if given in an otherwise dark field, represented the visual environment, would be correctly perceived, although these displacements took place, of course, only during head movements. Since these head movements cause relative environmental displacements of their own, the compensation process that is responsible for the perception of the objective displacements represents an interesting achievement.

Since the visual stimulation produced by a turning of the head can be identical with the stimulation produced by a rotation of the visual environment around a subject whose head is stationary, only proprioception of head movements or of immobility of the head can distinguish between the two conditions and produce correct visual perception. Since turning the head normally causes an equal angular displacement of the stationary environment in the opposite direction, an objective displacement of the environment during head turning merely adds to or subtracts from the angular displacement normally caused by the head movement. It is the detection of such additional or subtractive displacements that is responsible for the perception of the objective displacements which, caused by our apparatus, occur during head turning. This detection requires a match- ing up of the angular environmental displacement with the angle of the head rotation, and that is the task of the postulated compensation process.

This matching-up process is very accurate, as the detection of additional or subtractive displacements of the visual environment produced by the apparatus of Wallach and Kravitz has a very low threshold. Objective angular target displacement rates amounting to $2 \%$ of the rate of the head rotation are on the average correctly perceived. Since the objective displacement of the environment can be in two directions, either the same sense as the head rotation or in the direction against that of the head rotation, there are two thresholds of environmental displacement. The range of environmental displacements between these thresholds, where no environmental motions are perceived, was called the no-motion range. When sharp lines or contours were visible, the center of the no-motion range coincided accurately with objective immobility of the environment (Wallach \& Canal, 1976). ${ }^{1}$

The constancy of visual direction can be modified by adaptation to conditions where head movements are regularly accompanied by displacements of the visual eitvironment. Such an adaptation manifests itself in two ways: A stationary visual environment will appear to move during every head turning in a sense opposite to the displacements to which the subject had been adapted. Also, one can measure a no-motion range in Wallach and Kravitz' apparatus. It will coincide with some range of objective displacements of the environment in the same sense as the displacements present during the adaptation period. ${ }^{2}$ Either one of these facts can be used to measure adaptation. ${ }^{3}$ One can use the apparent motion of the stationary environment during head turning and obtain an estimate of its rate relative to the rate of the head rotation (estimation method), or one can measure the no-motion range after adaptation and compare it with the no-motion range before adaptation (compensation method) (Figure 1).

The measurements for the compensation method are obtained by presenting the subject with a series of different displacement ratios and by asking which ones of them led to perception of a stationary environment and which led to perceive motion. A displacement ratio is the amount of objective environmental displacement produced by a head movement of a particular extent or the rate of objective displacement divided by the rate of the head rotation. The displacement ratio (DR) is, then, the unit of measurement of the constancy of visual direction. We gave it as a percentage of environmental displacement rate over head-rotation rate, \%DR for short. Since the environmental displacement can be in either sense, in the direction with the head turning or in the direction against the head turning, the scale of DR values consists of diminishing environmental 


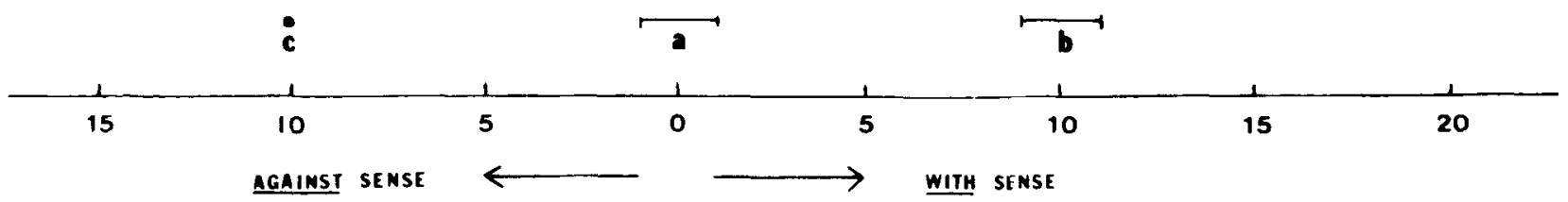

Figure 1. Scale of displacement ratios (target motion/head rotation), with 0 the objective no-motion point. Displacement ratios in the sense with the head rotation increase toward the right and target motion against the head rotation increases toward the left. $a$ is the no-motion range measured before adaptation. $b$ is the no-motion range measured after adaptation to displacements in the with sense by a compensation test. $c$ refers to the estimation test. It shows the displacement ratio of a target spot that is seen to move as much and in the same sense as a stationary spot appears to move after adaptation. The no-motion range $b$ can be thought of as compensating for this apparent motion.

displacements of one sense toward zero DR, which represents an objectively stationary environment, and of rising DR value in the other sense. In the preadaptation test, displacement ratios producing apparent immobility of the environment are distributed around zero DR. When adaptation is to environmental displacements in the direction with the head turning, as was the case in nearly all experiments to be reported here, the no-motion range obtained in the postadaptation test will comprise DR values in the with sense. If adaptation is complete, the no-motion range will be distributed around the DR value of the environmental displacements to which the subject had adapted. In the case of partial adaptation, the postadaptation no-motion range will comprise DR values that lie between that of the DR value of the adaptation condition and zero DR. The difference between the midpoints of the pre- and postadaptation no-motion ranges measures the amount of adaptation achieved. This procedure is called compensation method because the no-motion range measured after adaptation consists, in our present example, of environmental displacements in the direction with the head movements, which can be thought of as compensating for the apparent displacements of the stationary environment in the direction against the head movement.

When the estimation method is employed after adaptation to environmental displacements in the with direction, the subject perceives a stationary environment to move in the against sense. Here the amount of adaptation achieved is measured by obtaining an estimate of the DR equivalent to the perceived motion of the stationary environment. This was done directly by the slant estimation method developed by Wallach and Frey (1969). The indirect method employed in the present work consisted in obtaining an estimate of the extent of the apparent environmental motion produced by a head turning through a fixed angle.

The work to be reported below is concerned with a recent finding by Wallach and Canal, that two radically different conditions yield adaptation in CVD. In one condition, the subject fixated throughout the adaptation period, which he spent in Wallach and Kravitz' apparatus, a mark that was laterally displaced at $40 \% \mathrm{DR}$ in the sense with the subject's head-turning movements. But the remainder of the environment was not dark, as had been the case in previous work where adaptation had been to the displacements of a luminous spot, but was filled by a stationary pattern that subtended a visual angle of 48.5 deg. Wallach and Canal called the adaptation effect produced by this condition "eye-movement adaptation," because here adaptation was obviously caused by the abnormal eye movements that the subject made during his head movements. Compared to the normal compensatory eye movements that take place when, during head turning, one looks at a point in the stationary environment, the subject's eye movements were diminished, since the fixation mark was consistently displaced by $40 \% \mathrm{DR}$ in the direction with the head rotation. In the other condition, the large pattern was being displaced dependent on the head movement, but the subject was not allowed to follow these displacements with his eyes, as he had been free to do in previous CVD adaptation experiments; rather, he had to fixate, throughout the adaptation period, a stationary mark. As a result, he made only normal compensatory eye movements while he turned his head. Since this adaptation could have been caused only by the displacing pattern, we called the resulting effect "field adaptation."

The adaptation effects reported by Wallach and Canal were obtained with the compensation method. One of the aims of the present study was the development of an estimation method compatible with the new apparatus. We also meant to make another attempt to compare the results obtained with the estimation and the compensation method for identical adaptation conditions. The new apparatus provided for the projection of a patterned surface that could be displaced at various rates by the subject's head movements and filled his whole visual field.

Our report consists of two parts. The experiments reported in Part I serve to compare adaptation produced by three kinds of exposure: under free viewing conditions, under the conditions yielding eye- 
movement adaptation, and under those yielding field adaptation. For each kind of exposure, we used two kinds of tests, the compensation method and an estimation method. In Part II, the nature of eye-movement adaptation is investigated and experiments are reported that throw some light on the nature of field adaptation.

\section{PART I}

\section{Methods}

Equipment. Our new apparatus provided a pattern of vertical shadow bars that filled the subject's visual field and could be made to rotate about him. The rotation was arranged to be dependent on the subject's head turning by means of a variable ratio transmission. By adjusting the transmission, the experimenter could cause the shadow pattern to rotate in the sense with the head rotation or in the opposite sense, and the ratio of pattern displacement to head turning, the DR, could also be varied. Attached to a vertical steel column was a horizontal frame on which the variable ratio transmission was mounted so that its input shaft was vertical. Connected to the input shaft by a pair of gears was a secondary vertical shaft extending downward, to which a welder's headgear worn by the subject could be attached. The subject sat on an adjustable chair with his back to the steel column directly beneath the secondary shaft. The transmission was mounted in such a way that its horizontal output shaft pointed forward. Attached to it was a right-angle gear whose secondary shaft was vertical and approximately in line with the secondary input shaft. An arrangement of vertical rods forming a cylindrical cage rose from a circular plate, which was mounted on the vertical secondary output shaft. The 3-mm-diam rods were spaced $4 \mathrm{~mm}$. The cage, whose diameter was $15 \mathrm{~cm}$, was connected to the secondary output shaft by two retaining pins. When the experimental conditions called for the field to be stationary as the subject turned his head, the pins were removed and the cage was lifted by a sliding arm that disengaged it from the supporting shaft. A point source of light located at a point on the axis of the cylindrical cage furnished the illumination for the shadow pattern cast by the rods of the cage.

The gears between the primary and secondary input shafts served two purposes. They caused the latter, to which the subject's head was attached, to be approximately aligned with the axis of the cage, and they provided a rotation ratio of 5 to 1 between the secondary and the primary input shaft. This ratio served to make more effective use of the transmission. ${ }^{4}$ The control shaft of the transmission with which the transmission ratio was set and the rotation sense of the output shaft was altered was equipped with a Veeder counter, which provided readings of the transmission settings. The equation between the displacement ratios delivered by the apparatus at the various transmission settings and the counter readings was empirically determined.

This apparatus was surrounded by a large, nearly complete cylindrical screen, $200 \mathrm{~cm}$ in diameter and $192 \mathrm{~cm}$ high; there was an 80-deg gap in back of the transmission. The inner surface of the screen was lined with a homogeneous white Formica sheet and was concentric with the axis of the rod cage. The shadow pattern it cast on the screen thus filled the subject's visual field. ${ }^{5}$

Inside the cage, below the light source, was a mirror, which was mounted on an extension of the secondary output shaft. It served to reflect a narrow beam of light from a projection lantern that ended up on the screen in front of the subject approximately at the level of his eyes. This beam passed through the gap between two cage rods. It carried to the screen the projection of a small circular aperture with a thin vertical black line bisecting it. It formed a disk of light of $1 \mathrm{-cm}$ diameter on the screen, and, being in sharp focus, was an excellent target for accurate convergence and accommodation of the eyes. Being reflected by the mirror attached to the transmission's output shaft, it could, like the shadow of the cage, be made to displace dependent on the subject's head turning. The disk of light could also serve as a stationary fixation mark. For this purpose, it was projected by a second lantern that was directed toward a stationary mirror which again reflected its beam to a point on the screen in the center of the subject's field of vision.

Six different exposure conditions were used in which the subject adapted to pattern displacements that depended on his head movements. Subjects adapted to displacements either of a pattern that virtually filled their fields of vision or of a smaller pattern that was centered in the subject's field when his head position was straight ahead. The "small" field subtended an angle of $42 \mathrm{deg}$ and was produced by an aperture in a black cylinder that was slipped over the shadow casting cage when it was needed. Three modes of adaptation were employed: (1) The subject viewed the displacing pattern freely. Since this was the mode of exposure used in many of the adaptation experiments prior to those of Wallach and Canal, we called this mode "normal adaptation." (2) Only a small mark underwent displacements dependent on his head movements, which the subject had to keep fixating throughout, while either the large or the small pattern was visible and remained stationary (eye-movement adaptation). (3) The subject had to fixate a stationary mark, while either the large or the small pattern underwent displacements dependent on his head movements (field adaptation).

Because steady fixation of the stationary mark was very important in this last mode of presentation, a still smaller spot of light could be projected by a third lantern into the center of the stationary fixation mark at various times during the adaptation period. The subject's task was to press a switch whenever he saw the additional light appear in the mark. Since the additional light spot was very dim, it could be detected only when the subject was looking directly at the mark. This arrangement provided a constant check on the subject's proper fixation.

Under all conditions, the displacement of the pattern (or of the fixation mark when it was being displaced) amounted to $40 \%$ of the head rotation and was in the sense with the head rotation. The adaptation period always lasted $10 \mathrm{~min}$.

Two methods of measuring the adaptation effects were used because we had never demonstrated that the two tests were equivalent, and there appeared to be some chance that they were not. We also thought that the estimation test, which is by its very nature more rapid, might therefore measure larger adaptation effects.

The new estimation test. The slant estimation test that had been developed by Wallach and Frey (1969) could not be incorporated into the present apparatus. Instead, the apparent motion of a stationary target spot that resulted from adaptation was measured by obtaining an estimate of the distance through which it appeared to move during the turning of the head through a fixed angle. To provide for a head rotation through a fixed angle, a horizontal telescopic rod was attached to the secondary input shaft of the transmission. When the rod was pulled out, its end moved between two stops that limited the head rotation of $19 \mathrm{deg}$. After the subject had turned his head back and forth twice between these stops while looking at a stationary spot, a dim lamp was turned on, and he was given a paper pad on which he had to mark the distance through which this target had appeared to move.

The following procedure was used to evaluate the performance with which each subject expressed his estimate of the distance of the apparent target displacement. Before exposure to the adaptation condition, the subject was presented with a number of actual displacements of the target. In each case, he had to do the standard head turning and give an estimate in the manner described. Since adaptation was to displacements in the sense with the head movement, which would cause after adaptation an apparent displacement of a stationary target in the sense against the head movement, the actual target displacements used to calibrate the subject's estimation performance was in the sense against the head turning. Target displacements amounting to 
$0,2,4,6,8,12,16$, and $20 \% \mathrm{DR}$ were presented for estimation in randomized order. The evaluation of the postadaptation estimate was based on this series of estimates in the following manner. It was assumed that the relationship between the eight preadaptation estimates was linear. This assumption was valid, since it was found that for every subject the correlation between the eight estimates and the objective displacements was very high; $r$ ranged from .80 to 98 . This made it possible to evaluate the postadaptation estimate in terms of the regression equation derived from the eight preadaptation êstimates. The preadaptation score that best represents that objective rarget displacement that is seen by an individual subject as stationary (as expressed by the subject's preadaptation estimates) is the y-intercept computed from the regression of the subject's displacement estimates ( $Y)$ on the objective displacements (X). This y-intercept thus makes use of al! eight preadaptation estimates a subject had given. It amounts to the displacement equivalent of the implicit immobility estimate of a subject. In order to transform the subject's postadaptation estimate into an objective displacement that best represents it, the slope and the $x$-intercept of the regression of $X$ on $Y$ was calculated. From the slope $m$ and the $\mathrm{X}$-intercept $\mathrm{b}$, the objective displacement $(\mathrm{X})$ that corresponds to a subject's estimate $(Y)$ can be computed by using the equation $\mathrm{X}=\mathrm{mY}+\mathrm{b}$. The difference between this displacement equivalent of the subject's postadaptation estimate and his preadaptation score is the subject's adaptation score. This procedure largely eliminates the variance that would result from the individual differences in the way subjects represent their estimates of target motion.

While the adaptation scores were based on a postadaptation estimate that followed the adaptation period immediately, 12 of our 16 subjects actually made two postexposure estimates. Following the one or two estimates, the subject was asked to close his eyes and sit, relaxed, without turning his head. After 10 min, all subjects gave another estimate. For 12 of our sub. jects, this was immediately followed by a second delayed estimate. These delayed estimates were meant to test for spontaneous dissipation of the adaptation effect. The 10 -min period during which the subject remained passive will be called the "dissipation period."

The compensation test. As stated, the compensation test measures two no-motion ranges, one before and the other after the adaptation period. The difference between the midpoints of the two ranges on the DR scale is the adaptation effect. Wallach and Kravitz $(1965,1968)$ used an abbreviated method of limits to measure the two thresholds of perceived motion that are the limits of the no-motion range. But since this method is slow, it seemed not suitable to measure the effects of brief adaptation periods, which may dissipate fairly rapidly. Wallach and Canal (1976) gave the control of the variable transmission to the subject and had him find the limits of the no-motion range, by changing the transmission ratio continuously at a slow rate. This method was rapid but perhaps influenced by the rate at which different subjects changed the displacement ratio. We used the rapid method of changing the DR continuously, but gave the experimenter control over the variable transmission. The experimenter initially set the DR to some value that produced displacement of the target spot in the same sense as the head movement and lowered it slowly at an even pace, while the subject turned his head back and forth and observed the target. As soon as the subject reported that the target no longer moved, the experimenter read the transmission setting with a dim flashlight. This reading constituted one limit of the subject's no-motion range. Then the experimenter set the transmission so that the subject saw the target move in the sense against the head rotation and gradually altered the DR in the direction opposite to the previous change, causing the subject to see slower target motion. A second reading taken when the target motion seemed to have just stopped was recorded as the other limit.

The limit of the no-motion range toward perceived target motion in the direction with the head rotation was always taken first and the other limit in the second place. This order made possible to keep exposure of the subject to target displacement that he saw as target motion in the sense against the head mo ement to a minimum. When it occurs in the postadaptation tesi, such an exposure could tend to diminish the effect of adaptation to environmental displacements in the sense with the head movements, which was produced in our experiments. Since this order was employed in both the pre- and the postadaptation test, it had no effect on the difference between their results. In finding the limits of the no-motion range, the experimenter initially set the transmission to a DR value likely to produce the proper motion report and made an adjustment in the case where the subject reported the target to appear stationary. Since he could not read the transmission setting without using a flashlight and since he stood so that he could not see the moving target, his conduct of the experiment could not influence the DR value of the subject's "no-motion" reports.

Because the compensation test takes more time than the estimation test, there was only a single postadaptation test. $\mathbf{R e}$ testing after a dissipation period was also omitted.

Two experiments were performed, one employing the estimation test and the other the compensation test. In the experiment employing the estimation test, the effects of six different adaptation conditions, three models in connection with two field sizes, were measured. The same 16 subjects were employed in the three modes done under whole-field conditions, and 15 of these subjects were used for the three conditions using the limited field. The order of the three modes was randomized within the whole-field condition and within the limited-field conditions. Half of the subjects adapted first to the whole-field and subsequently to the limited-field conditions, and for the other half this sequence was reversed. There was an interval of at least a week between each of the six experiments most subjects participared in.

The compensation test was used only under whole-field conditions. The same group of 28 subjects was used for eye-movement adaptation and for field adaptation. A different group of 12 subjects underwent normal adaptation.

\section{Results}

The mean adaptation effects for all nine conditions, six obtained with the estimation test and three with the compensation test, are listed in Table 1. An adaptation effect score is the difference between subject's pre- and postadaptation score expressed in $\% \mathrm{DR}$. (In the estimation test experiment, the mean adaptation effects presented in Table 1 were derived from the first postadaptation test.) Eight of the nine adaptation effects were significant at the .001 level; only in the case of field adaptation in the limited-field condition was $\mathrm{p}<.01$.

In the estimation test experiment, individual subjects served in six different conditions, but this fact did not affect the results. An analysis of variance showed that there was no order effect, $F(1,14)=016$. In this experiment, normal adaptation had much larger effects than eye-movement and field adaptation, in the whole-field as well as in the limited-field conditions; each of the four differences was significant at the .001 level. Field size did not affect the adaptation effects in the cases of normal and of eye-movement adaptation, but in the case of field adaptation the effect obtained with the limited field was significantly smaller than the one produced by the whole field $(2.5 \% \mathrm{DR}$ vs. $5.3 \% \mathrm{DR}), \mathrm{t}(14)=$ $3.8, \mathrm{p}<.01$. This is still found after the dissipation 
Table 1

Adaptation Effects of 10 Min Exposure to Three Modes of Adaptation and Two Field Sizes Measured With Two Tests

\begin{tabular}{|c|c|c|c|c|c|c|}
\hline \multirow[b]{2}{*}{$\begin{array}{l}\text { Mode } \\
\text { Test } \\
\text { Estimation }\end{array}$} & \multicolumn{3}{|c|}{ Whole Field } & \multicolumn{3}{|c|}{ Limited Field } \\
\hline & $\begin{array}{c}\text { Normal } \\
\text { Field Displaced } \\
\text { No Mark } \\
\end{array}$ & $\begin{array}{c}\text { Eye Movement } \\
\text { Field Stationary } \\
\text { Moving Mark }\end{array}$ & \begin{tabular}{l}
\multicolumn{1}{c}{ Field } \\
Field Displaced \\
Stationary Mark \\
\end{tabular} & Normal & Eye Movement & Field \\
\hline $\begin{array}{l}\text { Mean } \\
\text { SD } \\
t \\
N\end{array}$ & $\begin{array}{r}13.1 \\
6.8 \\
7.7 \\
16\end{array}$ & $\begin{array}{l}5.5 \\
2.5 \\
8.9 \\
16\end{array}$ & $\begin{array}{l}5.3 \dagger \\
3.0 \\
7.1 \\
16\end{array}$ & $\begin{array}{l}11.5 \\
4.3 \\
10.2 \\
15\end{array}$ & $\begin{array}{l}5.7 \\
3.4 \\
6.4 \\
15\end{array}$ & $\begin{array}{l}2.5 \dagger \dagger \\
2.5 \\
3.8^{*} \\
15\end{array}$ \\
\hline $\begin{array}{l}\text { Compensati } \\
\text { Mean } \\
\text { SD } \\
\text { t } \\
\mathrm{N}\end{array}$ & $\begin{array}{l}9.8 \\
3.7 \\
9.2 \\
12\end{array}$ & $\begin{array}{l}7.2 \\
2.8 \\
11.9 \\
28\end{array}$ & $\begin{array}{l}5.6 \\
2.5 \\
13.6 \\
28\end{array}$ & & & \\
\hline
\end{tabular}

${ }^{*} p<.01$

$+7.2 \%$ displacement ratio

† $4.7 \%$ displacement ratio

period. The adaptation effects obtained from the first test following the dissipation period are given as footnotes in Table 1. The effect was $7.2 \% \mathrm{DR}$ in the case of the whole field and 4.7 \%DR in the case of the limited field. The difference between the two effects was also significant, $\mathrm{t}(14)=2.48, \mathrm{p}<.05$. The unexpected and consistent increase in the adaptation effect after the dissipation period was peculiar to field adaptation; it did not occur in normal and in eye-movement adaptation.

The results obtained with the compensation test resembled those obtained with the estimation test in the whole-field condition in two ways: The mean effect measured for field adaptation was virtually the same as that obtained with the estimation test immediately after the adaptation period. The mean effects for normal adaptation were different, but the difference was not significant, $t(27)=1.67$. The results differed where eye-movement adaptation is concerned; it was significantly higher when measured with the compensation test, than when it was measured with the estimation test $(7.2 \% \mathrm{DR}$ vs. $5.5 \% \mathrm{DR}), \mathrm{t}(43)=2.17, \mathrm{p}<.05$. This results is confirmed when mean eye-movement adaptation is compared with mean field adaptation. Measured with the estimation test, they were virtually the same, but in the compensation test experiment, the mean effect of eye-movement adaptation was significantly greater than the mean field-adaptation effect. Because here the two means stem from the same large group of subjects, the significance was quite high, $\mathrm{t}(27)=3.79, \mathrm{p}<.001$. Why this difference emerged only when testing was with the compensation test remains unexplained.

The results for the second postadaptation and the second postdissipation test were remarkable in that, without exception, an estimation test that immediately followed an identical estimation test showed a smaller mean adaptation effect than the directly preceding test. Inasmuch as 12 such sequences occurred in our experiment, this finding is highly significant. Even more interesting is the result that in the cases of normal and of eye-movement adaptation, where no increase of the adaptation effect after the dissipation period occurred as had been found in the case of field adaptation, the mean effect measured in the first test after the dissipation period was greater than the mean effect obtained in the second postadaptation test. Since results to be reported below indicate that normal adaptation and eye-movement adaptation are functionally equivalent, we combined the results for these two conditions, those for the whole-field as well as those for the limited-field conditions, and subjected the overall means for the four sequential tests to an analysis of variance. The results are presented in Table 2. The overall means show the drops between the first and the second postadaptation test and between the two postdissipation tests; either one amounted to about $2 \% \mathrm{DR}$. The overall means also show that there were no dissipation effects. The effects obtained in the two tests after the dissipation period had almost exactly the same means as the corresponding means derived from the two postadaptation tests. The recovery of the adaptation effect during the dissipation period amounted to $2.2 \% \mathrm{DR}$ and was highly significant. We believe that the drop between the first and the second of consecutive tests is due to a partial unlearning of the

Table 2

Overall Mean Effects of Normal and Eye Movement Adaptation Measured Twice With the Estimation Test Immediately After the Exposure Period and After a 10-Min Delay (Percent Displacement Ratio)

\begin{tabular}{lcc}
\multicolumn{1}{c}{ Tests } & Mean & $\begin{array}{c}\text { Fo of } \\
\text { Differences }\end{array}$ \\
\hline First Postadaptation & 8.95 & $15.1^{*}$ \\
Second Postadaptation & 6.90 & $17.3^{*}$ \\
First Postdissipation & 9.10 & $16.4^{*}$ \\
Second Postdissipation & 6.95 & \\
\hline
\end{tabular}

${ }^{*} p<.01$ 
adaptation, produced by the presentation of an objectively stationary target spot in the test. This stationary presentation, however, is very brief compared to the 10 -min adaptation period. It has an effect on the immediate following test, where it interferes with the effect of the adaptation training, only because of its recency. When this recency is lost during the 10-min interval of the dissipation period, the adaptation effect regains its original value.

Two conclusions regarding normal and eyemovement adaptation can be drawn from these findings. The interference-recovery pattern that emerged from our test sequence suggests that only the first postadaptation estimate be used to measure the adaptation effect. It also makes the compensation test preferable. The complete recovery of the adaptation effect after a 10-min interval removes our concern about the duration of the compensation test. Since this test avoids presentation of a stationary target spot, interference is probably kept to a minimum, particularly when testing always starts with target displacements in the same sense as the displacements to which the subject had been adapted. To this the fact must be added that the compensation test brackets the no-motion range, and an individual score, being the average of the two limits, more nearly represents the midpoint of the no-motion range than the single score in the single trial estimation test, which falls somewhere in the no-motion range.

The second conclusion was quite unexpected: A 10-min delay of the test after the end of the adaptation period caused ne dissipation of the adaptation effect. This result contradicts a finding by Wallach and Frey (1972), according to which an adaptation effect produced in about $8 \mathrm{~min}$ with a procedure then assumed to be normal decreased by $33 \%$ during a $10-\mathrm{min}$ dissipation period. We are now inclined to hold Wallach and Frey's particular adaptation procedure responsible for the discrepancy.

\section{PART II}

\section{The Nature of Eye-Movement Adaptation}

When under ordinary circumstances, that is, with the visual environment stationary, one looks at a particular point while turning one's head, the eyes make compensatory movements. They resemble pursuit movements in that the eyes remain directed on a displacing point and are slow compared to saccades. But a pursuit movement that occurs in response to a fixated object starting to move begins with a delay, while the compensatory eye movement that keeps the eye fixed on a point that becomes displaced due to a head movement starts immediately. The pursuit movement occurs in response to image displacement on the retina, the compensatory movement in response to the head movement (Woodworth, 1938). It then seems reasonable to assume that eye-movement adaptation is accompanied by modification of these compensatory eye movements.

The afterimage test. To test this hypothesis, we measured compensatory eye movements before and after the adaptation period with an afterimage test. The subject, whose head was in normal position, was asked to fixate a luminous spot straight in front of him and to turn his head to the left as soon as the spot disappeared, keeping his eyes on the place where the spot had been. A stop limited the head movement to $15 \mathrm{deg}$, and a switch activated a flashgun as soon as the stop was reached. The flashgun was just below the location of the spot and was masked except for a narrow vertical oblong opening coming to a tip at the upper end. The subject was asked to turn his head back to the normal position and the screen in front of him was lit, displaying a thin vertical straight line which the subject had to fixate. The experimenter held a vertical black cardboard strip that came to a tip at the lower end against the screen, and the subject directed him to move it so that its tip touched the tip of the afterimage that had emerged on the screen. The experimenter marked the location of the tip lightly on the screen. This mark represented the location of the afterimage relative to the fixated line.

The experiment began by having the subject go through this procedure four times. If three of the four marks that represented the location of the afterimage relative to the fovea were within $5 \mathrm{deg}$ of visual angle of each other, the subject was accepted for the experiment. He then underwent a standard 10-min period of eye-movement adaptation immediately followed by a single afterimage test (1). Because we could not prevent all head movements while the subject fixated the vertical line as he located the afterimage, it was possible that that test was an occasion for an interference with the adaptation effect. To overcome such a possible effect of the test, the subject was then given a further brief adaptation period of $1 \mathrm{~min}$ duration. This was followed by a compensation test. After another 1-min adaptation period, a second single afterimage test (2) was administered. This procedure was repeated after at least a week had elapsed, but now, as a control condition, the subject was exposed to field adaptation in the adaptation period. Five of our 10 subjects did the experiments in this order; for the other five, the order of experimental and control session was reversed.

Results of the afterimage tests. The mean adaptation scores obtained with the afterimage tests were computed in the following manner. The most deviant of the four afterimage locations obtained before the 
adaptation period was discarded, and the average of the remaining three locations was computed. The horizontal distance between this average and the afterimage location found in the postadaptation test was a subject's adaptation effect score. This adaptation effect score was transformed into its displacement ratio equivalent by computing the visual angle of the horizontal distance of which this score consisted and by dividing by 15 . (The flash, it will be remembered, always occurred after a head movement of 15 deg.) Since there were two postadaptation tests, there were two sets of adaptation effect scores and two mean adaptation effects. They are listed in Table 3 under 1 and 2 in the second and third columns. Since the mean adaptation effects measured with the first and second postadaptation afterimage test were virtually the same, the results of the postadaptation compensation test that were obtained after the first and before the second afterimage test seemed acceptable and were incorporated into the compensation test results given in Table 1. These results are presented again in the first column of Table 3.

Eye-movement adaptation to a displacement of the fixation mark with the head movement required a mark to be displaced on the average by $7.2 \% \mathrm{DR}$ with the head movement in order to appear stationary. To keep the eyes fixed on a mark that is being so displaced requires smaller eye movements. If eye-movement adaptation is accompanied by a modification of compensatory eye movements, the latter should be found to be shortened after such an adaptation, and that was the case. The results of the afterimage test in the second and third columns of Table 3 represent a significant shortening of eye movements that compensate for a head turning; the shortening amounted to $13 \% \mathrm{DR}$. No such effect was found after field adaptation, and the difference was significant at the .01 level. The latter result was to be expected since, during field adaptation, the subjects fixated a stationary mark and made normal compensatory eye movements. The difference between the change measured by the afterimage tests and the adaptation effect measured by the compensation test was not significant. It is, therefore, possible to assume that the change in compensatory eye movements measured after the adaptation period matches the adaptation effect.

What conclusion could one draw, were this certain? Could one say that eye-movement adaptation is based on a change in the compensatory eye movements that are performed during adaptation and test? While we have no data that permit an answer to this question, there are facts that have a bearing on a related one. In normal constancy of visual direction (CVD), is CVD a matter of compensatory eye movements, to the extent to which relative environmental displacements during head move-
Table 3

Adaptation (Percent Displacement Ratio) After Four Conditions of Adaptation With Five Different Methods of Testing

\begin{tabular}{|c|c|c|c|c|c|c|}
\hline \multirow{3}{*}{$\begin{array}{l}\text { Adaptation } \\
\text { Condition }\end{array}$} & \multicolumn{6}{|c|}{ Test Method } \\
\hline & \multirow{2}{*}{$\begin{array}{l}\text { Compen- } \\
\text { sation }\end{array}$} & \multicolumn{2}{|c|}{$\begin{array}{l}\text { After- } \\
\text { image }\end{array}$} & \multicolumn{2}{|c|}{ Pointing } & \multirow{2}{*}{$\begin{array}{c}\text { Porward } \\
\text { Direc- } \\
\text { tion }\end{array}$} \\
\hline & & 1 & 2 & I & II & \\
\hline Normal & 9.8 & & & 12.6 & & -.43 \\
\hline Eye Movement & 7.2 & 12.9 & 13.0 & 13.3 & 10.6 & -1.3 \\
\hline Field With Fixation & 5.6 & -.6 & 2.4 & .2 & & 8.7 \\
\hline Field With Saccades & & & & & & 17.2 \\
\hline
\end{tabular}

ments are given through eye movements? The relevant facts came from a comparison of the results of the preadaptation afterimage test with those of the preadaptation compensation test. We obtained a measure for the accuracy of normal compensatory eye movement by computing the average deviations of the eight initial image locations which each subject gave before the adaptation period in the two experimental sessions. Again, the mean of these measures was transformed into a displacement ratio equivalent by computing the visual angle of the mean average deviation and dividing by 15 . The transformed mean amounted to $4.2 \% \mathrm{DR}$. The accuracy of the CVD itself is measured by the no-motion range which, for the 10 subjects of the present experiment, had a mean of $2.32 \% \mathrm{DR}$. This value had to be halved to make it correspond to the mean average deviation. The difference between the two means $(4.2$ vs. $1.16 \% \mathrm{DR})$, was conspicuous, $\mathrm{t}(9)=3.8$, $\mathrm{p}<.01$. Further, the mean preadaptation afterimage location was $5.8 \% \mathrm{DR}$ to the right of where the flash had been; it was displaced in the direction opposite to the head movement. This displacement was significantly different from zero, $t(9)=3.17$, $\mathrm{p}<.02$. The mean midpoint of the no-motion range measured with the compensation test prior to adaptation was $1.0 \% \mathrm{DR}$ in the direction with the head movement. These two means were significantly different, $t(9)=3.5, p<.01$, and their variances also differed significantly $(p<.01)$. Probably the strongest indication that the two tests do not measure closely related functions results from the finding that the correlation between the subjects' performances in the two tests was virtually zero $(r=.05)$.

Whether one concludes from these results, as we tentatively do, that CVD cannot be based on compensatory eye movements depends on whether one accepts our afterimage test as an adequate device to measure the extent of compensatory eye movements. But even if this were in doubt, our main result, namely, that eye-movement adaptation is accompanied by a change in compensatory eye movements, would not be affected, inasmuch as any shortcoming of the afterimage test would have the same effect on the pre- and the postadaptation test.

The pointing test. The fact that a change in 
compensatory eye movements accompanies eyemovement adaptation cannot, under any circumstances, explain eye-movement adaptation fully. A change in the evaluation of compensatory eye movements must accompany their alteration. Only if the shortened compensatory eye movements produced by eye-movement adaptation were evaluated as normal could they account for adaptation in CVD. It should therefore be expected that after eyemovement adaptation the extent of compensatory eye movements should be overrated. Pointing Test I served to demonstrate such a change in the evaluation of compensatory eye movements. In total darkness, the subject turned his head $18 \mathrm{deg}$ to the left. As soon as his head movement stopped, a short vertical line straight in front of his body lit up, and the subject pointed at it without seeing his pointing hand. This test was given before and after the period of eye-movement adaptation. Overrating, due to adaptation, of the compensatory eye movement connected with a turning of the head to the left means that the subject should point farther to the right after the adaptation period than before.

There was again a control experiment in which subjects underwent field adaptation and were tested with the Pointing Test I. Different groups of subjects were employed for the two modes of adaptation. After we had found that, as expected, the pointing test registered only eye-movement adaptation, we also used it in connection with normal adaptation. We wanted to know the degree to which normal adaptation consisted in eye-movement adaptation.

To make the direction in which the subject pointed visible in the dark, he held in his hand a Bakelite tube, $2.5 \mathrm{~cm}$ in diam and $17.5 \mathrm{~cm}$ long, that was made into a small projector. A lens at one end projected a sharp image of the straight filament of a small bulb positioned inside the tube on the screen in front of the subject when he held it with outstretched arm. The experimenter could turn the bulb on and off. The subject held the tube in his right hand so that it was aligned with his pointing index finger. The tube was marked so that the subjects always held it in such a way that the projected luminous test line was vertical.

In the test, a vertical luminous target line was projected on the screen directly in front of the subject. With the pointing tube properly placed into his hand, the subject turned his head to the left until he touched a stop after an 18-deg turn. Immediately the target line lit up and the subject had to point at it. Keeping his arm in position, he closed his eyes. The experimenter lit the bulb in the pointing projector and marked the position of the test line on the screen. The average location of the test line relative to the target line of three such pointing trials was the sub- ject's preadaptation score. Subjects whose individual pointings were highly variable were eliminated at this time. There were another three pointing trials after adaptation, and their mean became the postadaptation score. Throughout the experiment, the subject held the pointing tube firmly. (The pointing trials were actually interspersed with trials of the FD test to be discussed below; no order effects could be discerned.)

Results of Pointing Test $I$. The mean differences between the pre- and postadaptation scores for the different adaptation modes were again changed into $\%$ DR measures and are so listed in the fourth column of Table 3. Of these mean adaptation effects, the one pertaining to eye-movement adaptation amounted to $13.3 \% \mathrm{DR}$ and was in good agreement with the adaptation effect measured with afterimage test (12.9 \%DR). As was expected, the pointing test did not measure field adaptation. A mean adaptation effect of .2\%DR was obtained which differed significantly from the eye-movement adaptation effect, $\mathrm{t}(22)=2.2$, $\mathrm{p}<.05$. The effect of normal adaptation measured with the pointing test amounting to $12.6 \% \mathrm{DR}, \mathrm{t}(11)=4.65, \mathrm{p}<.001$, was as large as the effect so measured for eye-movement adaptation. It seems that normal adaptation is eyemovement adaptation. Below, we shall find this conclusion confirmed.

The experiments with the afterimage test demonstrated that eye-movement adaptation involves changes in compensatory eye movements. As already stated, such changes in compensatory eye movements can explain adaptation only if the evaluation of these eye movements is also altered. Adaptation measured with Pointing Test I demonstrated such a changed evaluation, an overrating of compensatory eye movements as a result of adaptation. After discussing preadaptation measurements of compensatory eye movements that were obtained in the afterimage test, we concluded tentatively that CVD as such does not result from compensatory eye movements. This suggested to us that eye-movement adaptation, too, may not be based on changes in compensatory eye movements. Eye-movement adaptation may simply consist in a changed evaluation of all kinds of eye movements. Pointing Test II was designed to test this possibility. In this test, the subject pointed at a target after he had made a lateral eye movement that was not accompanied by a head movement.

At the start of the test, the subject sat with his head locked in the normal position and fixated a mark straight in front of him. The mark was extinguished and another spot $18 \mathrm{deg}$ to the right of the mark was turned on. The subject was instructed to look directly at the spot as soon as it appeared and then to point at it. The same pointing equipment was used as in Pointing Test $I$, and there were again three pre- 
adaptation and three postadaptation trials; the difference between their average results became a subject's adaptation effect score.

As had been the case in Pointing Test $I$, the eye movement to the right made by the subject just before the pointing in the postadaptation test had evidently been overrated, because the subjects pointed on the average $1.9 \mathrm{deg}$ farther to the right after adaptation than they had done before adaptation. This mean adaptation effect, obtained from 18 subjects and transformed as before amounted to $10.6 \% \mathrm{DR}$ and was highly significant, $t(17)=3.96$, $p<.001$. This result easily accounted for the effect of eye-movement adaptation measured with the compensation test of $7.2 \% \mathrm{DR}$.

The square test. It has often been claimed that eye movements play a part in the perception of shape. If this were true, shape perception could serve as another specific test for eye-movement adaptation, which, as we have seen, consists in a changed evaluation of eye movements. Moreover, if such a shape test is found to measure eye-movement adaptation, that is, if a change in the evaluation of eye movements caused by adaptation alters shape perception, a role of eye movements in shape perception would be firmly established. Since our eyemovement adaptation resulted in an overrating of horizontal eye movements only, giving subjects the task to change the width of an oblong until it appears to him square should serve as such a shape test.

Our equipment consisted of a light box with a milk-glass window, on which a changeable aperture frame was placed. Two pieces made up this aperture. One was a metal frame that covered the window and was firmly attached to it; its oblong opening was $12.5 \mathrm{~cm}$ high and $17 \mathrm{~cm}$ wide. In front of this frame piece was an oblong sheet of metal, higher than the aperture in the frame piece, that could slide horizontally between two horizonal bars attached to the frame piece. This sliding metal piece could be made to cover more or less of the oblong aperture in the frame piece. Its position was controlled by a long horizontal screw that turned in a nut attached to the light box. Its end rested against a block fixed to the sliding piece, with a gear mounted on the other end. A horizontal coil spring, one end attached to the light box and the other to the sliding piece, firmly pulled the latter against the end of the screw. The receiver of a pair of torque transmitters was mounted on the extended base of the light box so that a gear on its shaft engaged the gear on the end of the screw. The transmitter was equipped with a disk and a crank knob and was mounted near the subject's right hand. This arrangement enabled the subject to give to the lighted aperture a variety of shapes from an oblong resting on its side to a square and to a standing oblong. A horizontal scale mounted on the light box and a pointer attached to the sliding piece enabled the experimenter to read the aperture width after the subject had made a setting. He did this with a flashlight, since the tests were conducted in the dark. A second torque transmitter was available to the experimenter. By throwing a switch, he could take control of the receiver away from the subject's transmitter and give it to his own transmitter.

During testing, the subject's head was in a headrest. The adjustable aperture was $28 \mathrm{~cm}$ from his eyes. Its constant height of $12.5 \mathrm{~cm}$ subtended a visual angle of $25 \mathrm{deg}$.

In the case of one-half of the subjects, the experimenter set the aperture to a shape that was wider than a square. The subject had the task of turning the crank counterclockwise and thereby making the aperture narrower until it looked square to him. Then he closed his eyes and signaled to the experimenter, who read the setting he had made. With this descending trial over, the experimenter set the aperture so that it appeared clearly too narrow, and the ascending trial began; now the subject made the oblong look square by turning the crank clockwise. Two such pairs of descending and ascending trials were done before the adaptation period. The first pair was made for practice, while the average of the two settings produced in the second trial pair was the subject's preadaptation score. Another pair of settings, again starting with the descending trial, was made after the adaptation period. Their mean formed the postadaptation score. In the case of the other half of the subjects, each pair of settings started with an ascending trial. During the adaptation period, the subject was exposed to our eyemovement adaptation conditions, with the fixated mark being displaced at $40 \% \mathrm{DR}$ in the sense with the head turning.

There was a control condition where, during testing, the subject had to keep his eyes fixed on a stationary mark that was stuck to the milk glass visible in the aperture. Its location was slightly asymmetrical, $5 \mathrm{~cm}$ from the fixed vertical aperture edge and $7.2 \mathrm{~cm}$ above the lower horizontal edge. Different groups of 12 subjects each were employed in the experimental and the control conditions.

Results of the square test. Since our standard eyemovement adaptation caused horizontal eye movements to become overrated, shapes should, after the adaptation period, look wider than their actual width would warrant, that is, if eye movements do affect shape perception. Subjects should then be expected to set the aperture narrower after the adaptation period than before. Preventing eye movements during the tests by having the subject fixate a stationary mark, on the other hand, should prevent eye-movement adaptation from having an effect on the square test. Such results were, in fact, obtained.

The results of eye-movement adaptation tested 
Table 4

Adaptation Effect Measured With the Square Test

\begin{tabular}{|c|c|c|c|c|c|c|}
\hline \multicolumn{2}{|c|}{ Conditions } & \multirow[b]{2}{*}{$\mathrm{N}$} & \multirow[b]{2}{*}{ Effect } & \multirow[b]{2}{*}{$t$} & & \multirow[b]{2}{*}{$\mathrm{p}$} \\
\hline Adaptation & Test & & & & & \\
\hline Eye Movement* & $\begin{array}{l}\text { Without Fixation } \\
\text { With Fixation }\end{array}$ & $\begin{array}{l}12 \\
12\end{array}$ & $\begin{array}{l}4.5 \dagger \\
-.4\end{array}$ & 8.64 & n.s. & $<.001$ \\
\hline Magnifiers & Without Fixation & 12 & 1.9 & 5.13 & & $<.001$ \\
\hline Field With Fixation & $\begin{array}{l}\text { Without Fixation } \\
\text { With Fixation }\end{array}$ & $\begin{array}{l}12 \\
14\end{array}$ & $\begin{array}{l}3.0 \\
2.2\end{array}$ & $\begin{array}{l}5.29 \\
2.79\end{array}$ & & $\begin{array}{l}<.001 \\
<.02\end{array}$ \\
\hline Field With Saccades & With Fixation & 10 & 3.5 & 4.04 & & $<.001$ \\
\hline
\end{tabular}

Note-Mean differences in millimeters between the pre-and postadaptation shape settings.

${ }^{*}$ Difference $=4.9, t=5.8, p<.001$

with the square test are presented in the first two lines of Table 4 . When eye movements were permitted in the test, the mean difference between the pre- and postadaptation scores was $4.5 \mathrm{~mm}$, a change in mean width setting of $3.6 \%$, which was highly significant. In the control condition, there was no such difference; the corresponding mean amounted to $-.4 \mathrm{~mm}$, with the minus sign denoting a widening instead of a narrowing. The difference between the mean effects measured for the two adaptation conditions was highly significant $\mathrm{t}(22)=5.8, \mathrm{p}<.001$. We conclude that the square test measures an adaptation effect only when the subject is permitted eye movements in that test. This result, that the square test measured eye-movement adaptation only if the subject was allowed to make eye movements, confirms our view that eye-movement adaptation consists in a changed evaluation of eye movements. The result also indicates that eye movements can contribute to the perception of shape. But how important is that contribution? Our test figure was quite large as shapes go with which we are usually concerned; its height, which was not altered in the test, subtended an angle of $25 \mathrm{deg}$. When we increased the distance between the subject's eyes and the adjustable aperture from 28 to $110 \mathrm{~cm}$ so that its height subtended an angle of $6.5 \mathrm{deg}$ only, the square test measured no adaptation effect at all. ${ }^{7}$

The effect of eye-movement adaptation we measured with the large square test, amounting to $4.5 \mathrm{~mm}$, is equivalent to $3.7 \% \mathrm{DR}$. It was, thus, only half as large as the adaptation effect of $7.2 \% \mathrm{DR}$ measured with the compensation test, and the difference was significant at the .001 level, $\mathrm{t}(38)=5.4$. This difference is obviously a matter of the relative contributions that eye movements and image shape make to the perception of an oblong that subtends an angle of $25 \mathrm{deg}$.

Adaptation to normal displacement in the sense against the head movements measured with the square test. So far, our subjects had always adapted to a displacement in the sense with the head rotation amounting to $40 \% \mathrm{DR}$ and the adaptation period had been brief, namely, $10 \mathrm{~min}$. We thought it appropriate to measure with a square test an adaptation effect obtained under very different conditions. We used optical displacement of the environment caused by goggles that provided 2 power magnification. They cause an optical displacement of $100 \% D R$ in the sense against the head movement. ${ }^{8}$ To limit the subject's head movements to rotation about a vertical axis, he wore a headgear that was attached to a vertical shaft above his head. The subject faced a TV set $430 \mathrm{~cm}$ from his eyes. A spot attached to the center of its screen served as fixation mark. The subject watched a broadcast for $1 / 2 \mathrm{~h}$, turning his head back and forth while keeping his eye on the fixation mark.

Since the spot the subject fixated during head movements was optically displaced in the against sense, he made longer than normal compensatory eye movements during the adaptation period and learned to underrate his eye movements. The horizontal dimension of the large test oblong should therefore look shorter after adaptation than before, and the subject should, at that point, set it wider to make it appear square. This was indeed the result of our experiment. The mean adaptation effect was smaller than the one just reported, only $1.9 \mathrm{~mm}$, but highly significant, $\mathrm{t}(11)=5.13, \mathrm{p}<.001$. We propose that the smaller effect resulted from the restricted visual angle of the magnifiers. It amounted to $8 \mathrm{deg}$ and prevented the subject's head rotations from exceeding $8 \mathrm{deg}$. This means that, behind the magnifiers, the subject's eye never turned more than $16 \mathrm{deg}$, while the eye movements that contributed to the perception of the test oblong's shape amounted to about $25 \mathrm{deg}$.

Data on the horizontal/vertical illusion. The preadaptation settings made by our subjects show, of course, the horizontal/vertical illusion. The illusion that consists in overrating the height of figures was about normal for the square that subtended an angle of $6.5 \mathrm{deg}$. The mean width of the $12.5-\mathrm{cm}-$ high oblong when it looked square was $13.4 \mathrm{~cm}$. Therefore the illusion amounted to 7\%. For the square that subtended an angle of $25 \mathrm{deg}$, the illusion was significantly smaller, namely, $2.9 \% \pm 1.6 \%$, 
$\mathrm{t}(46)=2.56, \quad \mathrm{p}<.02$. When the preadaptation settings were made with fixation, the illusion was changed little; it amounted to $2.6 \% \pm 1.6 \%$ for the 25-deg square.

\section{Specific Effects of Field Adaptation}

As in the case of eye-movement adaptation, adaptation to field displacements during head movements was tested with a target spot that could be made to move dependent on head movements. As a result of adaptation, a target spot appeared stationary in the compensation test when it actually moved at some rate during head turning, in the sense with the head turning when adaptation had been to field displacement in the sense with the head turning. This means that displacements of the target spot relative to the head that are smaller than the relative displacements that a stationary point undergoes during a head movement are accepted as normal. Thus, field adaptation can be described as a changed rating of target displacements during head movements, an overrating in the case of adaptation to field displacements in the sense with the head movements.

The forward direction test. The question arose as to whether there are further manifestations of field adaptation, effects that do not require the combination with head movements as our CVD tests do. We already knew that field adaptation does not manifest itself in a pointing test. But it seemed possible that another directional test would work, one that operates on a different perceptual level from the pointing test. The forward direction (FD) test fits this description. It has been used as a test for adaptation to wedge prisms where the subject has the task to position in the dark a light point at eye level so that it appears straight in front of him. ${ }^{9} \mathrm{~A}$ test score is the average of a pair of settings obtained by having the subject direct the experimenter to shift the light point from the side toward the middle until it appears straight in front. One setting is made by starting the point too far to the right and the other by moving it in from the other side. In our use of the FD test, the subject had his head turned $18 \mathrm{deg}$ to the left and had the task to have a vertical target line $1 \mathrm{~cm}$ high and $.2 \mathrm{~cm}$ wide so positioned that it appeared to be straight in front of his body. The target line was projected on the curved screen that surrounded the subject throughout the experiment via a mirror that was mounted on a vertical shaft and could be turned by the experimenter by means of a set of reducing gears.

Initially, all our subjects performed very badly on this test. The interval between two of a pair of settings was large and the average of a pair of settings was highly variable. Our subjects were trained to do better by having them make their settings in general illumination that allowed them to see parts of their bodies. Also, each time a subject had made a setting, the target line was shifted into the correct position before his eye. After 10 or 15 min of this training, a subject was able to make two pairs of settings that were in good agreement with each other and whose average became his preadaptation score. Another two pairs of settings were made after the adaptation period, and their average became a subject's postadaptation score.

Twelve subjects were tested with this FD test in connection with field adaptation and another 12 in connection with eye-movement adaptation. Still another group of 12 subjects took the FD test in connection with normal adaptation. (As mentioned above, individual settings were actually alternated with the trials of Pointing Test I.)

Results of the FD test. The mean differences between the pre- and postadaptation scores obtained with FD tests were again transformed into $\% \mathrm{DR}$ and are so listed in the last column of Table 3 . A significant adaptation effect was obtained in connection with field adaptation. The mean postadaptation setting was $1.6 \mathrm{deg}$ farther to the right than the mean preadaptation setting, and this was equivalent to $8.7 \% \mathrm{DR}, \mathrm{t}(11)=4.2, \mathrm{p}<.01$. In the case of eye-movcment adaptation, the mean postadaptation setting was very slightly to the left of the preadaptation mean, and the difference between the two means was significant, $\mathrm{t}(11)=2.43, \mathrm{p}<.05$. The mean setting after normal adaptation showed a somewhat larger shift to the left amounting to $4.3 \% \mathrm{DR}$ which was, however, far from significant $(p=.2)$.

By measuring field adaptation and not eyemovement adaptation, the FD test can distinguish between the two modes of adaptation, just as the pointing test does by measuring eye-movement adaptation and not field adaptation.

The square test. We used this test in connection with field adaptation also. With the height of the oblong subtending a visual angle of $25 \mathrm{deg}$, the test measured an adaptation under both conditions, with movements permitted as well as with fixation. Here, too, horizontal distances looked slightly larger after the adaptation period than before, since, on the average, the objective shape that looked square was narrower after adaptation than it was before. The results are listed in the third and fourth line in Table 4. That the square test yields a measurement of field adaptation under fixation conditions also differentiates between field adaptation and eyemovement adaptation.

\section{Field Adaptation with Saccades}

The experiments just reported establish field adaptation as a process in its own right. It had an effect on perceived shape, whether observation is with or without eye movements, and it can be measured with a test that is specific to field adaptation, while there is still another test that measures 
only eye-movement adaptation. Prior to these findings, it was possible to consider eye-movement adaptation and field adaptation as procedures that ultimately have an effect on the same basic adaptation process. Now we have to regard them as two processes that are different in nature.

This conclusion raises a serious question about the nature of the normal constancy of visual direction (CVD). We have always assumed that CVD results from a long-range learning process, an adaptation to the ordinary displacement that the stationary environment undergoes relative to the head when the head is turned. (This permanent result of life-long adaptation becomes modified temporarily in experimental adaptation.) Is CVD, then, a combination of eye-movement adaptation and of field adaptation to such ordinary environmental displacements? The difficulty with that view is that conditions for field adaptation which we have employed thus far never occur under ordinary circumstances. The normal displacement of the stationary environment relative to the head that is caused by head turning is never given as an image displacement across the retina, that is, in a manner that would cause field adaptation. ${ }^{10}$ Does the fact that these conditions for field adaptation are never present when CVD is learned mean that field adaptation is merely an artifact of our experimental procedure, having no role in normal functioning? If that were the case, our finding that field adaptation is a learning product with properties of its own that can be measured by the same tests that measure eyemovement adaptation would be difficult to understand. Therefore we were not ready to accept the view that field adaptation could develop only in adaptation experiments. Instead, we searched for different conditions that could produce field adaptation, now defined as an adaptation that can be measured by the FD test and a square test with fixation.

Our search was based on the following considerations. We assumed that the learning that establishes CVD in everyone has a field adaptation component as well as an eye-movement adaptation component. Since we had come to the conclusion that our mode of normal adaptation consisted of eye-movement adaptation, we assumed that the learning that establishes CVD consists in eye-movement adaptation where it is based on the subject's keeping his eyes on a point in the visual environment while he turns his head. This is, however, only partly the case during ordinary head movements. When one turns one's head under ordinary circumstances, it is usually to look at another part of the visual field, and this involves saccadic eye movements in the direction of the head movement. Since saccades are faster than head movements, there are, during head movements, also periods during which the eyes are fixed on a point in the visual environment, and this involves slower, compensatory, eye movements. Learning to evaluate the latter so that the visual environment is perceived as stationary while they occur is the eyemovement adaptation component of the learning that establishes CVD.

We hypothesized that the field-adaptation component operates in connection with the saccadic eye movements that are made during head turning. Whether it occurs in connection with a head movement or not, a saccade brings into direct view a point in the visual field whose image has an extrafoveal position on the retina. But the eye movement that shifts the location of the point's image is not steered by the progress of that image across the retina. Rather, only the initial position of the point's image is responsible for the direction and the extent of the eye movement that brings it to the fovea. Hence, the properties of a saccade are determined by some representation of the visual field.

This nature of saccades makes more feasible the hypothesis that the field-adaptation component of the learning that establishes CVD is connected with saccades made during head movements. If this hypothesis is correct, it should be possible to show that a CVD adaptation condition where the subject makes frequent saccades during head movements produces field adaptation, namely, an adaptation that can be measured by the FD test and a square test with fixation.

To test this conclusion, our apparatus was modified to provide displacements of a letter display dependent on the subject's head turning. The cage of vertical rods and the small mirror were removed and a larger mirror $(26 \times 21 \mathrm{~cm})$ was mounted on the output shaft of the transmission. The slide that was projected onto the screen in front of the subject showed seven columns of letters, each consisting of 14 rows of three letters. The whole display subtended a visual angle of $33.5 \mathrm{deg}$, and the distance between columns subtended an angle of $2.5 \mathrm{deg}$. This display underwent displacements of $40 \% \mathrm{DR}$ in the sense with the head movement:

To induce the subject to perform under adaptation conditions that were like the natural combination of head movement and saccades described above, he was asked to read from left to right the letters in the first rows of the first and second columns while turning his head from left to right. He was then to turn his head to the left and move his eyes to the first letter of the second row of the first column and read the letters of that row in the first and second column during another head movement to the right. After reading the first two columns in this fashion, the subject continued in the same way with the third and fourth column, etc. Before and after this adaptation period, which lasted $10 \mathrm{~min}$ as always, each of the 12 subjects was given an FD test. 
As had been found in the original field-adaptation experiment, the mean postadaptation setting was farther to the right than the mean preadaptation setting. The difference amounted to $17.2 \% \mathrm{DR}$, $\mathrm{t}(11)=5.3, \mathrm{p}<.001$, and was nearly twice as large as the corresponding result obtained in the earlier experiment; the difference between the two results was significant at the .05 level.

When we repeated this experiment and measured with the square test, the effect we obtained was quite weak. We therefore revised the pattern of eye movements made in the adaptation condition so that it provided larger saccades. During head turning to the right, the subject had to read the rows of letters in the first, third, and fifth columns, then move his eyes to the second column during head turning to the left and read the rows in the second, fourth, and sixth column during the next head turning to the right, etc.

This adaptation procedure was quite successful. The square test with fixation showed that the shape of the oblong that looked square was $3.5 \mathrm{~mm}$ narrower after adaptation than before. This effect was significant at the .005 level, $t(9)=4.0$. It also differed significantly from the result obtained when the square test with fixation was used after eyemovement adaptation, $\mathrm{t}(20)=3.5, \mathrm{p}<.01$.

There are, then, two ways by which field adaptation, as defined by the specific tests that measure it, can be produced: under conditions of fixation of a stationary mark while the content of the visual field is being displaced dependent on head movements and under conditions of free observations of the displacing field involving frequent saccades. We call the latter condition "field adaptation with saccades." The former condition, which led to the discovery of field adaptation, will be called "field adaptation with fixation." The term "field adaptation" refers to the state in the nervous system which results from exposure to these conditions. ${ }^{11}$

Of the two tests that define field adaptation, the square test with fixation is the more important one. The FD test serves to distinguish field adaptation from normal and eye-movement adaptation, but it throws little light on its nature. The square test with fixation, on the other hand, tells us that field adaptation increases the perceived horizontal distance that corresponds to the retinal distance between the images of the two vertical lines, relative to the perceived vertical distance between the images of two horizontal lines. Field adaptation seems to alter the evaluation of retinal distances on which shape perception is based.

Although a change in the forward direction, such as is measured by the FD test, could be a manifestation of an altered evaluation of retinal distances, we do not believe that the results of the two tests are so closely related. The adaptation effects implied in the results of the two tests differ too much. Transformed into a DR equivalent, the change in the shape of the oblong caused by adaptation of $3.5 \mathrm{~mm}$ is equivalent only to $2.9 \% \mathrm{DR}$, while the corresponding value of the FD test was $17.2 \% \mathrm{DR}$. Similar differences are obtained when the effects of field adaptation with fixation are compared that are measured with the FD test and with the square test with fixation, namely, 8.7 \%DR vs. $1.8 \%$ DR. Unless a reason is found to explain why an altered evaluation of retinal distances can manifest itself only in small part in the square test, we have to assume that there are two components in field adaptation.

\section{CONCLUSION}

In Part I of this report, we described a new estimation test for the measurement of adaptation in CVD and presented results for three modes of adaptation and two field sizes. In the case of the large field size, the pattern filled the subject's whole visual field. Under the latter condition, compensation tests, too, were given in connection with the three modes of adaptation.

In Part II, we reported experiments which established that there are two different kinds of adaptation in CVD. One consists in an altered evaluation of eye movements and is produced when, during head movements, the eyes remain fixed on an objectively displacing spot and perform compensatory movements. The nature of the other kind, field adaptation, is not yet understood. It can result from displacement of the retinal projection of the whole field and it develops under normal adaptation conditions when saccadic eye movements are introduced. One possible interpretation, that field adaptation consists in a changed evaluation of head movements, can be rejected. A changed evaluation of head movement should manifest itself in a pointing test, but we reported that Pointing Test I measured no effect after field adaptation.

We propose the hypothesis that eye-movement adaptation and field adaptation take place at two different levels of visual processing, eye-movement adaptation at the level where eye positions are evaluated and field adaptation where the nervous system corrects for differences in head position as such. At the level where field adaptation takes place, the visual environment is represented as it is related to the head, that is, with the effect of eye position on retinal location already taken into account. Therefore, at this level, any eye-movement adaptation would have had its prior effect. It is the function of this level to take head movements and head position into account so that the visual environment appears stationary when, during head movements, the subject makes other than compensatory eye movements, and that visual directions can be correct- 
ly perceived when the head is turned out of the normal position. Field adaptation alters these compensating functions. Because saccadic eye movements during adaptation produce field adaptation, it seems likely that ordinary CVD is the result of two learning processes, one connected with compensatory eye movements and the other consisting in field adaptation to the normal displacements of the environment due to head movements, produced when saccadic eye movements occur.

Since we found that normal adaptation can be measured with a pointing test but not with an FD test, we assume that no eye movements that produce field adaptation occur in normal adaptation and that normal adaptation is essentially eye-movement adaptation. One might, therefore, expect to find that those two modes of adaptation produce effects in the same amount. This was, however, true only when the adaptation effect was measured with the pointing test (see Table 3). The two CVD tests showed a smaller effect after eye-movement adaptation than after normal adaptation. This difference was very large in the case of the estimation test (see Table 1), but it was significant also in the case of the compensation test, $\mathrm{t}(52)=2.18, \mathrm{p}<.05$.

We propose the following explanation for these seemingly contradictory results. The two CVD tests were the same for all three models of adaptation. These tests, thus, probe the state of the nervous system both with regard to eye-movement adaptation and with regard to field adaptation. This is not true of the pointing test which is, as we have found, specific to eye-movement adaptation. Therefore, if, after normal or eye-movement adaptation, the pointing test measured larger adaptation effects than a CVD test, ${ }^{12}$ it would be due to the fact that the CVD test also probes for the state of the nervous system with regard to field adaptation. Since no field adaptation developed under normal and under eyemovement adaptation conditions, as the results of the FD tests showed, the state of the nervous system after the adaptation period represented the ordinary CVD where field adaptation is concerned. This may have prevented the CVD tests from measuring the full eye-movement adaptation, as measured by the pointing test.

A similar consideration would explain the finding that the CVD tests show a larger effect after normal adaptation than after eye-movement adaptation. In the latter condition, not only do the eyes pursue the displacing target point, but a stationary pattern is also given. This stationary pattern, in undergoing the normal environmental displacements during head movements, provides training in the field-adaptation component of the ordinary CVD. It has been found on several occasions that training with the ordinary perceptual condition prior to adaptation training will diminish the effect produced by the latter
(Epstein, 1971; Wallach \& Floor, 1970; Wallach \& Karsh, 1963). The same should be true when training with the ordinary perceptual condition is simu:taneous with the adaptation training, as is the case in the eye-movement adaptation condition, which provides eye-movement adaptation training and at the same time strengthens the field-adaptation component of the ordinary CVD. Hence the difference between eye-movement adaptation and normal adaptation that was measured with the two CVD tests.

There were two incidental findings that are of general interest:

(1) An interference-recovery pattern after eyemovement adaptation was discovered when the estimation test was used in a search for spontaneous dissipation of the adaptation effect.

(2) Our result that eye-movement adaptation consists in a changed evaluation of eye movements makes it, for the first time, possible to gauge the relative contributions of image shape and eye movements to form perception. In the case of a square that subtended an angle of $25 \mathrm{deg}$, the contribution of eye movements was about $50 \%$. But with a square subtending an angle of $6.5 \mathrm{deg}$ only, still large where ordinary form perception is concerned, no contribution of eye movements could be detected. It seems safe to conclude that eye movements play only a minor role in form perception. Eye-movement adaptation, however, is now available as a tool for more detailed study.

\section{REFERENCE NOTE}

1. Whipple, W. R., \& Wallach, H. Direction-specific motion thresholds for abnormal image shifts during eye movements. Submitted for publication.

\section{REFERENCES}

Epstein, W. Adaptation to uniocular image magnification after varying preadaptation activities. American Journal of Psychology, 1971, 84, 66-74.

HAY, J. C. Visual adaptation to an altered correlation between eye movement and head movement. Science, 1968, 160, 429-430.

MACK, A. An investigation of the relationship between eye and retinal image movement in the perception of movement. Perception \& Psychophysics, 1970, 8, 291-297.

WALLACH, H., \& BACON, J. The constancy of the orientation of the visual field. Perception \& Psychophysics, 1976, 19, 375-382.

Wallach, H., \& Canal, T. Two kinds of adaptation in the constancy of visual direction. Perception \& Psychophysics, 1976, 19. 445-449.

Wallach, H., \& Flaherty, E. W. A compensation for field expansion caused by moving forward. Perception \& Psychophysics, $1975,17,445-449$.

Wallach, H., \& Floor, L. On the relation of field displacement during head movements to the constancy of visual direction. Perception \& Psychophysics, 1970, 8, 95-98.

W ALLACH. H., \& FreY, K. J. Adaptation in the constancy of visual direction measured by a one-trial method. Perception \& Psychophysics, 1969, 5, 249-252.

Wallach, H., \& Frey, K. J. Differences in the dissipation of the 
effect of adaptation to two kinds of field displacements during head movements. Perception \& Psychophysics, 1972, 11, 31-34.

WALLACH, H., \& KaRSH, E. G. Why the modification of stereoscopic depth perception is so rapid. American Journal of Psychology, 1963, 76, 413-420.

W allach, H., \& Kravitz, J. The measurement of the constancy of visual direction and of its adaptation. Psychonomic Science, $1965,2,217-218$.

Wallach, H. \& Kravitz, J. Adaptation in the constancy of visual direction tested by measuring the constancy of auditory direction. Perception \& Psychophysics, 1968, 4, 299-303.

Wallach, H., Kravitz, J. H., \& Lindauer, J. A passive condition for rapid adaptation to displaced visual direction. American Journal of Psychology, 1963, 76, 568-578.

Wallach, H., Stanton, L., \& BECKer, D. The compensation for movement-produced changes in object orientation. Perception \& Psychophysics, 1974, 15, 339-343.

Wallach, H., Yablick, G. S., \& Smrth, A. Target distance and adaptation in distance perception in the constancy of visual direction. Perception \& Psychophysics, 1972, 12, 139-145.

Woodworth, R. S. Experimental psychology. New York: Holt, 1938.

\section{NOTES}

1. Environmental displacements relative to the eyes that are due to head movements are greater than normal when the target is near. Wallach, Yablick, and Smith (1972) demonstrated that the compensating process takes target distance into account. Sharp lines and contours furnish better cues for target distance.

2. One can conceive of the range of objective displacements after adaptation as compensating for the apparent motion of the stationary environment.

3. Measuring the amount of adaptation that has developed makes it possible to experiment with partial adaptation, which can be produced in brief adaptation periods.

4. Decreasing the rotation rate of the primary input shaft relative to the head rotation caused the transmission to operate better at low transmission ratios.

5. We are grateful to Otto Hebel for constructing this apparatus.
6. This view differs from one proposed by John Hay (1968). He believes that a change in compensatory eye movements is sufficient to explain adaptation in CVD: "To the extent that the eyes' new movements keep the retinal image stationary there is no longer any visual stimulus for the perception of an object moving." Hay fails to specify that this simple principle can possibly apply only to compensatory eye movements, for pursuit eye movements do lead to perception of object motion, while the retinal image is stationary. At any rate, results of ours yet to be reported refute Hay's explanation. We shall present evidence that eye-movement adaptation does indeed involve a changed evaluation of eye movements.

7. A figure subtending a visual angle of $6.5 \mathrm{deg}$ measures about 1 in. across at 25 -cm viewing distance.

8. A point in the environment located in the median plane of the subject's head will, after a rotation of the head to the right by $1 \mathrm{deg}$, lie $1 \mathrm{deg}$ to the left of the changed median plane. Since 2 power magnification doubles all visual angles, the point, if seen through the magnifiers, will reach the eyes from a direction $2 \mathrm{deg}$ to the left of the changed median plane. It will thus have been displaced by $100 \% \mathrm{DR}$ from its original forward direction. The magnifiers were Galilean telescopes and were constructed in our laboratory.

9. The form in which the FD test is used here was first employed by Wallach, Kravitz, and Lindauer (1963).

10. In the original learning of CVD, the analogue to field adaptation would consist of a condition where one fixates a mark that shifts during head turning so that it remains always in one's median plane, while the stationary environment undergoes its ordinary displacements relative to the head. In such an arrangement, the normal environmental displacements due to head movements would be given as image displacements on the retina.

11. No pointing test has been performed after field adaptation with saccades, but it is reasonable to assume that this condition produces eye-movement adaptation also, since compensatory eye movements occur along with saccades.

12. This was indeed the case, but because of high variance in the pointing test results, the differences were not significant.

(Received for publication May 21, 1976; revision accepted November $30,1976$. 